\title{
On the Complexity of UC Commitments ${ }^{\star}$
}

\author{
Juan A. Garay ${ }^{1}$, Yuval Ishai ${ }^{2, \star \star}$, Ranjit Kumaresan ${ }^{2}$, and Hoeteck Wee ${ }^{3, \star \star \star}$ \\ 1 Yahoo Labs \\ garay@yahoo-inc.com \\ 2 Department of Computer Science, Technion, Haifa, Israel \\ \{yuvali,ranjit\}@cs.technion.ac.il \\ 3 ENS, Paris, France \\ wee@di.ens.fr
}

\begin{abstract}
Motivated by applications to secure multiparty computation, we study the complexity of realizing universally composable (UC) commitments. Several recent works obtain practical UC commitment protocols in the common reference string (CRS) model under the DDH assumption. These protocols have two main disadvantages. First, even when applied to long messages, they can only achieve a small constant rate (namely, the communication complexity is larger than the length of the message by a large constant factor). Second, they require computationally expensive public-key operations for each block of each message being committed.

Our main positive result is a UC commitment protocol that simultaneously avoids both of these limitations. It achieves an optimal rate of 1 (strictly speaking, $1-o(1)$ ) by making only few calls to an ideal oblivious transfer (OT) oracle and additionally making a black-box use of a (computationally inexpensive) PRG. By plugging in known efficient protocols for UC-secure OT, we get rate-1, computationally efficient UC commitment protocols under a variety of setup assumptions (including the CRS model) and under a variety of standard cryptographic assumptions (including DDH). We are not aware of any previous UC commitment protocols that achieve an optimal asymptotic rate.

A corollary of our technique is a rate- 1 construction for $U C$ commitment length extension, that is, a UC commitment protocol for a long message using a single ideal commitment for a short message. The extension protocol additionally requires the use of a semi-honest (stand-alone) OT protocol. This raises a natural question: can we achieve UC commitment length extension while using only inexpensive PRG operations as is the case for stand-alone commitments and UC OT? We answer this question in the negative, showing that the existence of a semi-honest OT protocol is necessary (and sufficient) for UC commitment length extension. This shows, quite surprisingly, that UC commitments are qualitatively different from both stand-alone commitments and UC OT.
\end{abstract}

* Research received funding from the European Union's Tenth Framework Programme (FP10/2010-2016) under grant agreement no. 259426 ERC-CaC.

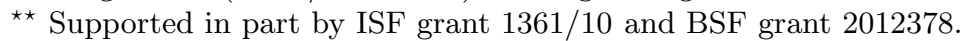

$\star \star \star$ CNRS (UMR 8548) and INRIA. Part of this work was done at George Washington University, supported by NSF Award CNS-1237429.

P.Q. Nguyen and E. Oswald (Eds.): EUROCRYPT 2014, LNCS 8441, pp. 677-694, 2014.

(C) International Association for Cryptologic Research 2014 
Keywords: Universal composability, UC commitments, oblivious transfer.

\section{Introduction}

A commitment scheme is a digital analogue of a locked box. It enables one party, called the committer, to transfer a value to another party, called the receiver, while keeping it hidden, and later reveal it while guaranteeing to the receiver its originality. Commitment schemes are a fundamental building block for cryptographic protocols withstanding active adversarial attacks. As such, efficient implementations of the latter-particularly in realistic complex environments where they are to execute - crucially hinge on them. Such complex environments are today epitomized by the universal composability (UC) framework [6], which allows for a protocol to run concurrently and asynchronously with arbitrarily many others, while guaranteeing its security.

The first constructions of UC commitments were given by Canetti et al. $7 / 8$ as a feasibility result. (It was also shown in [7] that it is impossible to construct UC commitments in the plain model, and that some setup such as a common reference string (CRS) is required.) Since then, and motivated by the above, a series of improvements (e.g., 141328 19 33|4|125]) culminated in constructions achieving under various cryptographic assumptions constant communication rate and practical computational complexity, making it possible to commit to, say, $L$ group elements by sending $O(L)$ group elements and performing $O(L)$ public-key operations (e.g., exponentiations).

Shortcomings - as well as ample room for improvement, however, remain, as the constant rate currently achieved is small and the computational cost per committed bit is high. This is the case even when committing to long messages and even when ignoring the cost of offline interaction that does not depend on the committed message. More concretely, the communication complexity is bigger than the length of the message by a large constant factor, and the online computation includes a large number of computationally expensive public-key operations for each block of the message being committed 1 This is not satisfactory when considering concrete applications where UC commitments are used, such as UC secure computation and UC zero-knowledge. (See 4281] for additional motivation on these applications.)

Our Results. We obtain both positive and negative results on the complexity of UC commitments. Our main positive result is a UC commitment protocol which simultaneously overcomes both of these limitations. Specifically, it achieves an optimal rate of 1 (strictly speaking, $1-o(1)$ ) by making only few calls to an ideal oblivious transfer (OT) oracle and additionally making a black-box use

${ }^{1}$ Recent constructions 2814 that work over standard DDH groups require at least 10 group elements and at least 20 public key operations per commitment instance. A very recent work by [25] (improving over [16]) requires 5 group elements in a bilinear group (assuming SXDH). 
of a (computationally inexpensive) PRG. By plugging in known efficient protocols for UC-secure OT (e.g., 34]), we get rate-1, computationally efficient UC commitment protocols under a variety of setup assumptions (including the CRS model) and under a variety of standard cryptographic assumptions (including DDH). We are not aware of any previous UC commitment protocols which achieve an optimal asymptotic rate.

Our main idea is to use a simple code-based generalization of the standard construction of commitment from $\delta$-Rabin-string-OTs [1126 2418]. The key observation is that the use of a rate- 1 encoding scheme with a judicious choice of parameters yields a rate- 1 construction of $\mathrm{UC}$ commitments.

Next, we show how to further reduce the computational complexity of the basic construction by using OT extension [2 2324]. Our improvement ideally suits the setting where we need to perform a large number of commitments in a single parallel commit phase (with potentially several reveal phases), as with applications involving cut-and-choose. In particular, we show that the number of calls to the OT oracle can be made independent of the number of instances of UC commitments required. (Note that such a result does not follow from multiple applications of the basic construction.) We stress that when handling a large number of commitment instances (say, in garbled circuit applications of cut-and-choose), the number of public key operations plays a significant role (perhaps more than the communication) in determining efficiency. While current state-of-the-art UC commitment protocols 28,4 suffer from the need to many computationally expensive public-key operations, our result above enables us to obtain better computational as well as overall efficiency.

Lastly, another corollary of our technique is a rate-1 construction for $U C$ commitment length extension, that is, a UC commitment protocol for a long message using a single ideal commitment for a short message. The extension protocol additionally requires the use of a semi-honest (stand-alone) OT protocol. This raises a natural question of whether we can achieve UC commitment length extension while using only inexpensive PRG operations as is the case for stand-alone commitments and UC OT. We answer this question in the negative, showing that the existence of a semi-honest OT protocol is necessary (and sufficient) for UC commitment length extension. This shows that UC commitments are qualitatively different from both stand-alone commitments and UC OT, which can be extended using any PRG [2], and are similar to adaptivelysecure OT whose extension requires the existence of (non-adaptively secure) oblivious transfer 29 .

We note that our constructions are only secure against a static (non-adaptive) adversary; we leave the extension to adaptive security for future work.

Related Work. We already mentioned above the series of results leading to constant-rate UC-commitments. Here we give a brief overview. Canetti et al. [78] were the first to construct (inefficient) UC commitments in the CRS model from general assumptions, and also achieve adaptive security. Shortly thereafter, Damgård and Nielsen [14] presented UC commitments with $O(1)$ exponentiations for committing to a single group element. Their construction is based on 
$N$-residuosity and $p$-subgroup assumptions, and is also adaptively secure (without erasures), but requires a CRS that grows linearly with the number of parties. A construction of Damgård and Groth [13, also adaptively secure without erasures and based on the strong RSA assumption, requires a fixed-length CRS.

An important improvement in concrete efficiency was presented recently by Lindell [28]; this is achieved for static corruptions based on the DDH assumption in the CRS model. Blazy et al. 4] build on Lindell's scheme to achieve adaptive security (assuming erasures); they also obtain improvements in concrete efficiency. Fischlin et al. [16] also build on Lindell's scheme and present a non-interactive scheme using Groth-Sahai proofs [21]. Furthermore, they also provide an adaptively secure variant (with erasures) based on the DLIN assumption on symmetric bilinear groups. As mentioned above, none of these works achieve rate 1 . We provide a concrete analysis of our protocol, with a comparison to 28,4 in Section 3.3 .

A code-based construction of UC commitments from OT was recently used by Frederiksen et al. [18] as part of an efficient protocol for secure two-party computation. While this construction uses a similar high level technique as our basic construction, its suggested instantiation in [18] only achieves a small constant rate.

Our work also considers the extension of UC commitments. We mainly focus on the goal of length extension, namely using an ideal commitment to a short string for implementing a UC commitment to a long string. For standalone commitments, such a length extension is easy to implement using any PRG. This is done similarly to the standard use of a PRG for implementing a hybrid encryption scheme. It was previously shown by Kraschewski[27] that this simple extension technique does not apply to UC commitments. We strengthen this negative result to show that any extension protocol for UC commitments implies oblivious transfer. Similar negative results for adaptively secure OT extension were obtained by Lindell and Zarosim [29]. and for reductions between finite functionalities by Maji et al. 30. Negative results for statistical UC coin-tossing extension were obtained by Hofheinz et al. 22 .

In an independent work [12, Damgård et al. also construct UC commitments using OT, PRG and secret sharing as the main ingredients. While the basic approach is closely related to ours, the concrete constructions are somewhat different, leading to incomparable results. In particular, a major goal in [12 is to optimize the asymptotic computational complexity as a function of the security parameter, achieving in one variant constant (amortized) computation overhead for the verifier. Moreover, they achieve both additive and multiplicative properties for UC commitments, which are not considered in our work.

Organization of the Paper. The rest of the paper is organized as follows. Model, definitions and basic functionalities are presented in Section 2, Our main construction - rate-1 UC commitment from OT-is presented in Section 3, together with the case of multiple commitment instances and a concrete efficiency analysis. Finally, the treatment of UC commitment extension-rate- 1 construction and necessity of OT - is presented in Section 4. Due to space limitations, 
only proof sketches are presented in the main body; full proofs as well as complementary material are deferred to the full version.

\section{Model and Definitions}

In this section we introduce some notation and definitions that will be used throughout the paper. We denote the computational security parameter by $\kappa$, and the statistical security parameter by $\sigma$. A function $\mu$ is negligible if for every polynomial $p$ there exists an integer $N$ such that for every $n>N$ it holds that $\mu(n)<1 / p(n)$.

In this paper we will be concerned with efficient universally composable (UC) [] realizations of functionalities such as commitments. Assuming already some familiarity with the framework, we note that it is possible to consider variants of the definition of UC security in which the order of quantifiers is " $\forall \mathcal{A} \exists \mathcal{S} \forall \mathcal{Z}$ ". Contrast this with our definition (and also the definition in [28]) in which the order of quantifiers is " $\exists \mathcal{S} \forall \mathcal{Z} \forall \mathcal{A}$ ". Both definitions are equivalent as long as $\mathcal{S}$, in the former definition makes only a blackbox use of $\mathcal{A}[6]$. Indeed, this will be the case in our constructions. Therefore, as in [28, we demonstrate a single simulator $\mathcal{S}$ that works for all adversaries and environments, and makes only a blackbox use of the

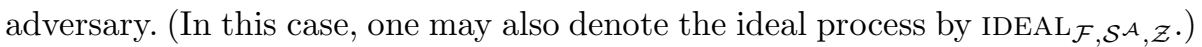

We will sometimes explicitly describe the functionalities we realize. For instance, if a functionality $\mathcal{F}$ accepts inputs only of a certain length $\ell$, then we will use the notation $\mathcal{F}[\ell]$ to denote this functionality. We let $\operatorname{cc}(\mathcal{F})$ denote the communication cost, measured in bits, of realizing $\mathcal{F}$ in the plain model.

The multi-commitment ideal functionality $\mathcal{F}_{\mathrm{MCOM}}$, which is the functionality that we UC realize in this work, is given in Figure 1. As mentioned above, $\mathcal{F}_{\mathrm{MCOM}}[\ell]$ will explicitly denote that the functionality accepts inputs of length exactly $\ell$. We will be giving our constructions in the OT-hybrid model.

\section{Functionality $\mathcal{F}_{\mathrm{MCOM}}$}

$\mathcal{F}_{\text {MCOM }}$ with session identifier sid proceeds as follows, running with parties $P_{1}, \ldots, P_{n}$, a parameter $1^{\kappa}$, and an adversary $\mathcal{S}$ :

- Commit phase: Upon receiving a message (commit, sid, ssid, $s, r, m$ ) from $P_{s}$ where $m \in\{0,1\}^{\ell}$, record the tuple $($ ssid, $s, r, m)$ and send the message (receipt, sid, ssid, $s, r$ ) to $P_{r}$ and $\mathcal{S}$. (The length of the strings $\ell$ is fixed and known to all parties.) Ignore any future commit messages with the same ssid from $P_{s}$ to $P_{r}$.

- Decommit phase: Upon receiving a message (reveal, sid, ssid) from $P_{s}$ : If a tuple (ssid, $s, r, m)$ was previously recorded, then send the message (reveal, sid, ssid, $s, r, m$ ) to $P_{r}$ and $\mathcal{S}$. Otherwise, ignore.

Fig. 1. Functionality $\mathcal{F}_{\mathrm{MCOM}}$ for multiple commitments 


\section{Functionality $\mathcal{F}_{\mathrm{OT}}^{N}$}

$\mathcal{F}_{\mathrm{OT}}^{N}$ with session identifier sid proceeds as follows, running with parties $P_{1}, \ldots, P_{n}$, a parameter $1^{\kappa}$, and an adversary $\mathcal{S}$ :

- Upon receiving a message (sender, sid, ssid, $s, r, x_{1}, \ldots, x_{N}$ ) from $P_{i}$, where each $x_{j} \in\{0,1\}^{\ell}$, record the tuple ( sid, ssid, $s, r, x_{1}, \ldots, x_{N}$ ). (The length of the strings $\ell$ is fixed and known to all parties.) Ignore any future sender messages with the same sid, ssid pair from $P_{s}$ to $P_{r}$.

- Upon receiving a message (receiver, sid, ssid, $s, r, q$ ) from $P_{r}$, where $q \in[N]$, send (sid,ssid, $\left.s, r, x_{q}\right)$ to $P_{r}$ and (sid,ssid,s,r) to $P_{s}$, and halt. (If no (sender, sid, ssid, $s, r, \ldots$ ) message was previously sent, then send nothing to Pr.)

Fig. 2. Functionality $\mathcal{F}_{\mathrm{OT}}^{N}$ for 1 -out-of- $N$ oblivious transfer. We omit superscript $N$ when $N=2$.

\section{Functionality $\mathcal{F}_{\mathrm{OT}_{\mathrm{R}}}^{\delta}$}

$\mathcal{F}_{\mathrm{OT}_{\mathrm{R}}}^{\delta}$ with session identifier sid proceeds as follows, running with parties $P_{1}, \ldots, P_{n}$, parameters $1^{\kappa}$ and a real number $\delta, 0<\delta<1$, and an adversary $\mathcal{S}:$

- Upon receiving a message (sender, sid, ssid, $s, r, x)$ from $P_{s}$, where $x \in\{0,1\}^{\ell}$, record the tuple (sid,ssid, s, r, x). (The length of the strings $\ell$ is fixed and known to all parties.)

- Upon receiving a message (receiver, sid, ssid, $s, r$ ) from $P_{r}$, set $y=x$ with probability $\delta$, and $y=\perp$ with probability $1-\delta$. Send (sid, ssid, $s, r, y)$ to $P_{r}$ and (sid, ssid, $s, r$ ) to $P_{r}$, and halt. (If no (sender, sid, ssid, $s, r, \ldots$ ) message was previously sent, then send nothing to $P_{r}$.)

Fig. 3. Functionality $\mathcal{F}_{\mathrm{OT}_{\mathrm{R}}}^{\delta}$ for Rabin-OT with noise rate $\delta$

The oblivious transfer functionality $\mathcal{F}_{\mathrm{OT}}^{N}$, capturing 1-out-of- $N$ OT for $N \in \mathbb{Z}$, is described in Figure 2. When $N=2$, this is the standard 1-out-of-2 string-OT functionality, denoted by $\mathcal{F}_{\mathrm{OT}}$. The $\delta$-Rabin-string-OT functionality, denoted $\mathcal{F}_{\mathrm{OT}_{\mathrm{R}}}^{\delta}$, is described in Figure 3 .

\section{Rate-1 UC Commitments from OT}

A recent line of work has focused on the practical efficiency of UC commitment in the CRS model 284119/33. In these works, a $\kappa$-bit string commitment is 
implemented by sending $O(1)$ group elements and computing $O(1)$ exponentiations in a DDH group of size $2^{O(\kappa)}$. We start this section by presenting a $\kappa$-bit UC-secure string commitment protocol in the $\mathcal{F}_{\text {OT }}$-hybrid model where the total communication complexity of each phase (including communication with the OT oracle) is $\kappa(1+o(1))$. The above implies that if OT exists (in the plain model), then there is a UC-secure protocol for an $N$-bit string commitment in the CRS model which uses only $N+o(N)$ bits of communication.

Thus, our construction improves over previous protocols which achieve constant rate, but not rate 1 . Using, for example, the DDH-based OT protocol of [34], we can get a rate-1 UC-commitment protocol in the CRS model which is quite efficient in practice; alternatively, if we wish to obtain a construction in the single global CRS model, we may instead start with the OT protocols given in [101]. We then address the setting where multiple UC commitments need to be realized, showing again a rate-1 construction where, in particular, the number of calls to the OT oracle is independent of the number of UC commitments required. We conclude the section with concrete efficiency analysis of our constructions.

On the "Optimality" of Our Construction. We note that our construction achieves essentially "optimal" rate. In any statistically binding commitment scheme as with our construction, the commit phase communication must be at least the message size. Moreover, any static UC secure commitment scheme must be equivocable, since the simulator for an honest sender does not know the message during the commit phase, and yet must be able to provide openings to any message. Therefore the communication in the decommit phase must be at least the message size, via an argument similar to the lower bound on secret key size in non-committing encryption 32 .

\subsection{Main Construction}

Our idea is to use a simple code-based generalization of the standard construction of commitment from $\delta$-Rabin-string-OTs [11262418]. Our key observation is that the use of a rate- 1 encoding scheme with a judicious choice of parameters yields a rate- 1 construction of UC commitments. We start off with the following reduction.

Rate-1 Rabin-OT from OT. We first show an efficient realization of Rabin-OT for a given $\delta \in(0,1)$, denoted $\mathcal{F}_{\mathrm{OT}_{\mathrm{R}}}^{\delta}$, in the $\mathcal{F}_{\mathrm{OT}}$-hybrid model, making black-box use of a PRG.

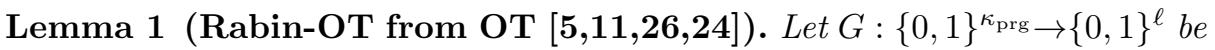
a secure $P R G$, and let $\delta \in(0,1)$ such that $1 / \delta$ is an integer. Then, there exists a protocol which $U C$-realizes a single instance of $\mathcal{F}_{\mathrm{OT}_{\mathrm{R}}}^{\delta}[\ell]$ in the $\mathcal{F}_{\mathrm{OT}}\left[\kappa_{\mathrm{prg}}\right]$-hybrid model such that:

- The protocol has total communication complexity at most $\ell+(1 / \delta)+3 \kappa_{\mathrm{prg}} \cdot 1 / \delta$ bits, including communication with $\mathcal{F}_{\mathrm{OT}}\left[\kappa_{\mathrm{prg}}\right]$. 
- The protocol makes at most $1 / \delta$ calls to the $\mathcal{F}_{\mathrm{OT}}\left[\kappa_{\mathrm{prg}}\right]$ functionality and requires each party to make a single invocation of $G$.

The protocol works by implementing $\mathcal{F}_{\mathrm{OT}_{\mathrm{R}}}^{\delta}[\ell]$ in the $\mathcal{F}_{\mathrm{OT}}^{N}[\ell]$-hybrid model for $N=1 / \delta$. Then $\mathcal{F}_{\mathrm{OT}}^{N}[\ell]$ is realized in the $\mathcal{F}_{\mathrm{OT}}\left[\kappa_{\mathrm{prg}}\right]$-hybrid model.

Rate-1 UC-Commitments from Rabin-OT. The construction is presented in the following lemma. Further construction and proof details can be found in the full version.

\section{Encoding scheme Enc}

Parameters: $n^{\prime}, d, n$ such that $n^{\prime}>d>n$.

Input: $m \in\{0,1\}^{\ell}$ for any $\ell>n \log \left(n+n^{\prime}\right)$.

- Parse $m \in\{0,1\}^{\ell}$ as $\left(m_{1}, \ldots, m_{n}\right) \in \mathbb{F}^{n}$ where $\mathbb{F}$ is such that $\log |\mathbb{F}|=\ell / n$.

- Let $e_{1}, \ldots, e_{n}$ and $\alpha_{1}, \ldots, \alpha_{n^{\prime}}$ be $\left(n+n^{\prime}\right)$ distinct elements in $\mathbb{F}$.

- Pick random polynomial $p$ of degree $d$ such that $m_{i}=p\left(e_{i}\right)$ for all $i \in[n]$.

- Output encoding $m^{\prime}=\left(p\left(\alpha_{1}\right), \ldots, p\left(\alpha_{n^{\prime}}\right)\right) \in \mathbb{F}^{n^{\prime}}$.

Fig. 4. A rate-1 encoding scheme based on the multi-secret sharing scheme of [17]

Lemma 2. Let $\sigma$ be a statistical security parameter, and let $n$ be such that there exists $\epsilon \in(0,1 / 2)$ satisfying $n^{1-2 \epsilon}=\sigma^{\Omega(1)}$. Then, for $\delta=\left(2 n^{\epsilon}+4\right)^{-1}$, and any $\ell>n \log \left(2 n+2 n^{1-\epsilon}\right)$, there exists a protocol that statistically $U C$ realizes a single instance of $\mathcal{F}_{\mathrm{MCOM}}[\ell]$ in the $\mathcal{F}_{\mathrm{OT}_{\mathrm{R}}}^{\delta}[\ell / n]$-hybrid model in the presence of static adversaries such that:

- The protocol has communication complexity $\ell\left(1+2 n^{-\epsilon}\right)$ bits in each phase, including communication with $\mathcal{F}_{\mathrm{OT}_{\mathrm{R}}}^{\delta}[\ell / n]$.

- The protocol makes $n\left(1+2 n^{-\epsilon}\right)$ calls to the $\mathcal{F}_{\mathrm{OT}_{\mathrm{R}}}^{\delta}[\ell / n]$ functionality.

Proof. The protocol uses the randomized encoding scheme Enc described in Figure 4 with parameters $n$ as in the Lemma, and $n^{\prime}=n+2 n^{1-\epsilon}$ and $d=$ $n+n^{1-\epsilon}-1$. Note that $\delta=(d+1-n) / 2 n^{\prime}$. Scheme Enc takes as input $m \in$ $\{0,1\}^{\ell}$ and parses them as $n$ elements from a field $\mathbb{F}$ and satisfies the following properties:

- it has rate $1+2 n^{-\epsilon}$;

- any $(d+1-n) / n^{\prime}=2 \delta$ fraction of the symbols reveal no information about the encoded message 2 ;

- any encodings of two distinct messages differ in $\Delta \stackrel{\text { def }}{=} n^{\prime}-d$ positions (and we can efficiently correct $\Delta / 2$ errors);

\footnotetext{
${ }^{2}$ We actually require a slightly stronger property to achieve equivocation, namely, that we can efficiently extend a random partial assignment to less than $2 \delta$ fraction of the symbols to an encoding of any message.
} 
The construction realizing $\mathcal{F}_{\mathrm{MCOM}}[\ell]$ in the $\mathcal{F}_{\mathrm{OT}_{\mathrm{R}}}^{\delta}[\ell / n]$-hybrid model is described in Figure 5. We first analyze the protocol's complexity:

Communication. In the commit phase, the sender transmits the encoding, i.e., $n\left(1+2 n^{-\epsilon}\right)$ symbols of $\mathbb{F}$ via $\mathcal{F}_{\mathrm{OT}_{\mathrm{R}}}^{\delta}[\ell / n]$. Since $\log |\mathbb{F}|=\ell / n$, the communication complexity is $\left(n+2 n^{1-\epsilon}\right) \cdot \ell / n=\ell\left(1+2 n^{-\epsilon}\right)$ bits. In the reveal phase, the sender sends the encoding in the clear. It follows from the calculations above that the communication complexity of this phase is also $\ell\left(1+2 n^{-\epsilon}\right)$ bits.

Computation. In the commit phase, the sender makes $n\left(1+2 n^{-\epsilon}\right)$ calls to $\mathcal{F}_{\mathrm{OT}_{\mathrm{R}}}^{\delta}[\ell / n]$.

We now turn to the proof of security. Note that $\delta=O\left(n^{-\epsilon}\right)$ while $\Delta=$ $O\left(n^{1-\epsilon}\right)$. Simulating when no party is corrupted or both parties are corrupted is straightforward. We briefly sketch how we simulate a corrupted sender and a corrupted receiver:

Corrupt Sender. Here the simulator extracts the committed value by looking at the corrupted codeword $\mathbf{c}$ that $P_{s}$ sends to the ideal OT functionality and compute the unique codeword $\mathbf{c}^{*}$ that differs from $\mathbf{c}$ in at most $\Delta / 2$ positions. In addition, the simulator reveals each symbol of $\mathbf{c}$ to the honest receiver with probability $\delta$. If $\mathbf{c}$ and $\mathbf{c}^{*}$ agree on all the positions that are revealed, then the committed value is the message corresponding to $\mathbf{c}^{*}$; else the committed value is $\perp$.

Next, suppose $P_{s}$ sends a codeword $\mathbf{c}^{\prime}$ in the reveal phase. We consider two cases:

- if $\mathbf{c}^{\prime}$ and $\mathbf{c}$ differ in at most $\Delta / 2$ positions, then $\mathbf{c}=\mathbf{c}^{*}$ and the simulator extracted the correct value;

- otherwise, the honest receiver accepts with probability at most $(1-\delta)^{\Delta / 2}$, which is negligible in $\sigma$.

Corrupt Receiver. In the commit phase, the simulator acts as the ideal OT functionality and for each symbol of the encoding, decides with probability $\delta$ whether to send (and, thereby fix) a random element of $\mathbb{F}$ as that symbol to the receiver.

Next, the simulator receives the actual message $m$ in the reveal phase. We consider two cases:

- As long as less than a $2 \delta$ fraction of the symbols are transmitted in the simulated commit phase above, the simulator can efficiently extend a random partial assignment implied by the transmitted symbols to the encoding of $m$;

- otherwise, the simulation of the reveal phase fails with probability at most $e^{-n^{\prime} \delta / 3}$, which is negligible in $\sigma$.

Putting things together:

Theorem 1 (Rate-1 UC commitments from OT). Let $\kappa$ be a computational security parameter, and let $\alpha \in(0,1 / 2)$. Then, there is a protocol which $U C$-realizes a single instance of $\mathcal{F}_{\mathrm{MCOM}}[\kappa]$ using $\kappa^{\alpha}$ calls to $\mathcal{F}_{\mathrm{OT}}\left[\kappa^{\alpha}\right]$ and a black-box use of a PRG, where the total communication complexity of each phase (including communication with $\mathcal{F}_{\mathrm{OT}}$ ) is $\kappa(1+o(1))$. 
Realizing $\mathcal{F}_{\mathrm{MCOM}}$ in the $\mathcal{F}_{\mathrm{OT}_{\mathrm{R}}}^{\delta}$-hybrid model

Let Enc : $\mathbb{F}^{n} \rightarrow \mathbb{F}^{n^{\prime}}$ be a randomized encoding scheme as in Figure 4

\section{Commit Phase.}

1. Upon receiving input (commit, sid,ssid, $s, r, m$ ) with $\ell$-bit input $m$, party $P_{s}$ parses $m$ as $\left(m_{1}, \ldots, m_{n}\right) \in \mathbb{F}^{n}$. It then computes $m^{\prime}=$ $\left(m_{1}^{\prime}, \ldots, m_{n^{\prime}}^{\prime}\right) \leftarrow \operatorname{Enc}(m)$.

2. For each $j \in\left[n^{\prime}\right]$ :

- $P_{s}$ sends (sender, sid, ssid $\circ j, s, r, m_{j}^{\prime}$ ) to $\mathcal{F}_{\mathrm{OT}_{\mathrm{R}}}^{\delta}$.

- $\quad P_{r}$ sends (receiver, sid, ssid $\left.\circ j, s, r\right)$ to $\mathcal{F}_{\mathrm{OT}_{\mathrm{R}}}^{\delta}$.

- $\quad P_{s}$ and $P_{r}$ receive $(s i d, s s i d \circ j, s, r)$ and $\left(s i d, s s i d \circ j, s, r, y_{j}\right)$ respectively from $\mathcal{F}_{\mathrm{OT}_{\mathrm{R}}}^{\delta}$.

3. $\quad P_{s}$ keeps state ( sid, ssid, $\left.s, r, m, m^{\prime}\right)$.

4. $\quad P_{r}$ keeps state (sid, ssid, $s, r,\left\{y_{j}\right\}_{j \in\left[n^{\prime}\right]}$ ), and outputs (receipt, sid, ssid, $s, r$ ). Also, $P_{r}$ ignores any later commitment messages with the same (sid, ssid) from $P_{s}$.

\section{Opening Phase.}

1. Upon input (reveal, sid, ssid, $P_{s}, P_{r}$ ), party $P_{s}$ sends $\left(\right.$ sid, ssid, $m^{\prime}$ ), where $m^{\prime} \in \mathbb{F}^{n^{\prime}}$, to $P_{r}$. Let $P_{r}$ receive $\left(\right.$ sid, ssid, $\left.\widetilde{m}^{\prime}\right)$, where $\widetilde{m}^{\prime}=\left(\widetilde{m}_{1}^{\prime}, \ldots, \widetilde{m}_{n^{\prime}}^{\prime}\right)$.

2. Let $J$ denote the set $\left\{j: y_{j} \neq \perp\right\}$. $P_{r}$ outputs $\perp$ if any of the following checks fail:

- $\quad \widetilde{m}^{\prime}$ is an (error-free) codeword;

- for all $j \in J$, it holds that $y_{j}=\widetilde{m}_{j}^{\prime}$.

If both conditions hold, $P_{r}$ decodes $\widetilde{m}^{\prime}$ to obtain $\widetilde{m}$, and outputs (reveal, sid, ssid, $s, r, \widetilde{m}$ ).

Fig. 5. A statistically UC-secure protocol for $\mathcal{F}_{\mathrm{MCOM}}$ in the $\mathcal{F}_{\mathrm{OT}_{\mathrm{R}}}^{\delta}$-hybrid model

Proof. We set $\ell=\kappa$ and $\sigma=\kappa$. Then we pick $n, \epsilon \in(0,1 / 2)$ such that $n^{1+\epsilon}=$ $\kappa^{\alpha} / 10$. Note that $\sigma, n, \epsilon, \ell$ satisfy conditions of Lemma 2 . Further, setting $\kappa_{\text {prg }}=$ $\kappa^{\alpha}$, also ensures that $O\left(\kappa_{\mathrm{prg}} n^{1+\epsilon}\right)=o(\kappa)$. The security proof readily follows from composing the protocols given in the Lemmas 1 and 2, We just need to analyze the complexity of the resulting protocol.

Communication. By Lemma 1 to implement $n+2 n^{1-\epsilon}$ calls to $\mathcal{F}_{\mathrm{OT}_{\mathrm{R}}}^{\delta}[\kappa / n]$, we need to communicate $\left(n+2 n^{1-\epsilon}\right)\left((\kappa / n)+O\left(\kappa_{\text {prg }} n^{\epsilon}\right)\right)=\kappa+2 \kappa n^{1-\epsilon}+O\left(\kappa_{\text {prg }} n^{1+\epsilon}\right)$ bits in the $\mathcal{F}_{\mathrm{OT}}\left[\kappa_{\mathrm{prg}}\right]$-hybrid model. For $n, \epsilon, \kappa_{\text {prg }}$ as set above, it follows that the communication cost of this phase is $\kappa(1+o(1))$ bits in each phase. Computation. By Lemma 1, to implement the required $n+2 n^{1-\epsilon}$ calls to $\mathcal{F}_{\mathrm{OT}_{\mathrm{R}}}^{\delta}[\kappa / n]$, we need to make blackbox use of PRG, and additionally $\left(n+2 n^{1-\epsilon}\right) \cdot(1 / \delta)=2 n^{1+\epsilon}+8 n$, i.e., at most $\kappa^{\alpha}$ calls to the $\mathcal{F}_{\text {OT }}\left[\kappa^{\alpha}\right]$ functionality. 


\subsection{Multiple Commitment Instances}

Next, we show how to further reduce the computational complexity of the previous construction by using OT extension 22324. Our improvement here extends to the setting where we need to perform a large number of commitments in a single parallel commit phase (with potentially many reveal phases), as with applications involving cut-and-choose. In particular, we show that the number of calls to $\mathcal{F}_{\mathrm{OT}}\left[\kappa_{\mathrm{prg}}\right]$ can be made independent of the number of instances of UC commitments required. (Note that such a result does not follow from multiple applications of the protocol implied by Theorem 1)

Theorem 2. Let $\kappa$ be a computational security parameter, and let $\alpha \in(0,1 / 2)$. For all $c>0$, there exists a protocol which UC-realizes $\kappa^{c}$ instances of $\mathcal{F}_{\mathrm{MCOM}}[\kappa]$ with rate $1+o(1)$ that makes $\kappa^{\alpha}$ calls to $\mathcal{F}_{\mathrm{OT}}\left[\kappa^{\alpha}\right]$ and a blackbox use of correlation robust hash functions (alternatively, random oracle, or non-blackbox use of oneway functions).

Proof. We repeat the protocol of Theorem $1 \kappa^{c}$ times to construct $\kappa^{c}$ instances of $\mathcal{F}_{\mathrm{MCOM}}[\kappa]$ using $\kappa^{c+\alpha}$ calls to $\mathcal{F}_{\mathrm{OT}}\left[\kappa^{\alpha}\right]$. By Theorem 1 the communication cost of this construction is $\kappa^{c}(1+o(1))$. We note that for each instance of this protocol, the commit phase has $o(\kappa)$ communication in addition to the cost involved in communicating with $\mathcal{F}_{\mathrm{OT}}\left[\kappa^{\alpha}\right]$.

We then implement the required $\kappa^{c+\alpha}$ calls to $\mathcal{F}_{\mathrm{OT}}\left[\kappa^{\alpha}\right]$ using the constant rate UC-secure OT extension protocol of 24] which makes blackbox use of correlation robust hash functions (alternatively, random oracle, or non-blackbox use of oneway functions). This implementation requires $\kappa^{\alpha}$ calls to the $\mathcal{F}_{\mathrm{OT}}\left[\kappa^{\alpha}\right]$ functionality, and has communication complexity $O\left(\kappa^{c+2 \alpha}\right)=o\left(\kappa^{c+1}\right)$ bits for $\alpha \in(0,1 / 2)$. Therefore, the total communication complexity of this protocol in each phase (including communication with the $\mathcal{F}_{\mathrm{OT}}\left[\kappa^{\alpha}\right]$ functionality) is $\kappa^{c}(1+o(1)$ ) for $c>1$.

\subsection{Concrete Efficiency Analysis}

In this section, we provide an analysis of the concrete efficiency of our proto$\mathrm{col}$, specifically requiring that the statistical security loss be $<2^{-\sigma}$ for statistical security parameter $\sigma$, and the seedlength for PRG be 128 . This reflects the state-of-the-art choices for similar parameters in implementations of secure computation protocols. In addition to the communication complexity, we will also be interested in the number of public key operations. (In practice, public-key operations (e.g., modular exponentiation) are (at least) 3-4 orders of magnitude slower than symmetric-key operations (e.g., AES).)

In the concrete instantiation of our UC commitment protocol in the CRS model, we will use (1) the protocol of Nielsen et al. 31] for OT extension in the $\mathrm{RO}$ model since it has better concrete security ( cost $\approx 6 \cdot 128$ bits for each instance of 128-bit OT excluding the "seed" OTs) than the protocol of [24]), and (2) the protocol of Peikert et al. 34 to realize "seed" OTs in the CRS model (with concrete cost per OT instance equal to 5 modular exponentiations and 6 elements 
in a DDH group of size 256). Note that for realizing 128 instances of $\mathcal{F}_{\mathrm{OT}}$ [128], the cost is $6 \cdot 128 \cdot 256=196608$ bits and the number of modular exponentiations is $5 \cdot 128=6403$ We stress that this cost is independent of parameters $\ell, \sigma$, and number of commitment instances. In the following we summarize the cost of our construction for some parameters. Our costs are calculated by choosing concrete parameters for the encoding scheme Enc used in Lemma 2, and then apply the transformation of Lemma 1, and finally realizing $\mathcal{F}_{\mathrm{OT}}$ using state-ofthe-art protocols as discussed above.

For long strings, say of length $\ell=2^{30}$, and for $\sigma=30$, we can get concrete rate as low as $1.046^{-1}$ in each phase. However, the choice of parameters necessitate working over a field $\mathbb{F}$ with $\log |\mathbb{F}|=2^{19}$. If we work over relatively smaller fields $\mathbb{F}$ with say $\log |\mathbb{F}|=512$, then the rate of the encoding can be made $1.19^{-1}$ (resp. $2.01^{-1}$ ), but the cost of realizing OTs (including OT extension) makes the total rate of the commit phase $\approx 9.58^{-1}$ (resp. $5.55^{-1}$ ). Note, however, that there are standard techniques to reduce the communication cost of realizing OTs in our setting. For instance, by replacing Rabin-OT with $d$-out-of- $n^{\prime}$ OT (for $d, n^{\prime}$ as in Figure (4), we may then use standard OT length extension techniques. This however has the drawback that RS encodings need to be performed over large fields, and further the number of public-key operations increases with the number of commitment instances.

Consider the following alternative approach that ports our construction to work with smaller fields, and yet get concrete rate close to 1 . First, the sender parse the message $m$ as a matrix where each element of the matrix is now from the field of desired size. Next, the sender performs a row-wise encoding (using Enc) of this matrix, and sends each column of the encoded matrix via $\mathcal{F}_{\mathrm{OT}_{\mathrm{R}}}^{\delta}$. Later in the reveal phase, the sender simply transmits the encoded matrix. As noted earlier, the above approach lets us work over small fields, and the concrete rate would be as good as the concrete rate for encoding each row.

Next, we discuss the cost of our basic construction when committing to short strings. For short strings, say of length $\ell=512$ (resp. 256) and $\sigma=20$, while the rate of our reveal phase can be as low as $4.6^{-1}$ (resp. 8.12-1), the rate of our commit phase can be very high $\left(\approx 1000^{-1}\right)$. While we concede that this is not very impressive in terms of communication cost, we wish to stress that our constructions do offer a significant computational advantage over the protocols of [28]4] since we perform only a fixed number of public key operations independent of the number of commitment instances. In Appendix $\mathrm{A}$, we propose efficient constructions to handle commitments over short strings in settings where a large number of such short commitments are used, e.g., in cut-and-choose techniques.

Efficiency in the preprocessing model. Our protocols can be efficiently adapted to the preprocessing model [331, and further, the online phase of our protocol can

\footnotetext{
${ }^{3}$ The protocol of 34] requires CRS of size $m$ for $m$ parties (cf. 10]). However, since CRS is a one-time setup, this does not affect our (amortized) communication cost. Alternatively, we could use the DDH based construction of 10 which uses a constant sized (6 group elements) global CRS for all parties and will only mildly increase (by a multiplicative factor $\approx 6$ ) the cost of realizing the "seed" OTs).
} 
be made free of cryptographic operations. First, note that any UC commitment protocol can be preprocessed, for example by committing to a random string in the offline model, and sending the real input masked with this random string in the online commit phase. Therefore, the online rate of the commit phase of the protocol in the preprocessing model can always be made 1 . Next, the online rate in the reveal phase of our protocol is exactly the rate of the underlying encoding. Note that in the online reveal phase, we only need the receiver to check the validity of the encoding.

\section{UC Commitment Extension}

As a corollary of our technique above, we start this section by showing a rate1 construction for $U C$ commitment length extension, that is, a UC commitment protocol for a long message using a single ideal commitment for a short message. The extension protocol additionally requires the use of a semi-honest (stand-alone) OT protocol. We then show that the existence of a semi-honest $\mathrm{OT}$ protocol is necessary for UC commitment length extension.

\subsection{Rate-1 UC Commitment Length Extension}

In this setting, we want a secure realization of a single instance of UC commitment on a $\ell$-bit string, for $\ell=\operatorname{poly}(\kappa)$, while allowing the parties to access ideal functionality $\mathcal{F}_{\mathrm{MCOM}}[\kappa]$ exactly once. We show that UC commitment length extension can be realized with rate $1-o(1)$.

Theorem 3 (Rate-1 UC commitment length extension). Let $\kappa$ be a computational security parameter, and assume the existence of semi-honest standalone oblivious transfer. Then, for all $c>0$, there exists a protocol which $U C$-realizes a single instance of $\mathcal{F}_{\mathrm{MCOM}}\left[\kappa^{c}\right]$ with rate $1-o(1)$ and makes a single call to $\mathcal{F}_{\mathrm{MCOM}}[\kappa]$.

Proof. The desired protocol is obtained by using the results of [15]9] to implement the necessary calls to the OT functionality in a protocol obtained by composing protocols of Lemma 2 and Lemma 1.

Using a single call to $\mathcal{F}_{\mathrm{MCOM}}[\kappa]$, we can generate a uniformly random string (URS) of length $\kappa$. Interpreting this $\kappa$-bit string as a $\kappa^{1 / 2}$ instances of a $\kappa^{1 / 2}$-bit URS, and assuming the existence of semi-honest stand-alone OT, one can apply the results of Damgård et al. [15, or Choi et a. 9] to obtain $\kappa^{\alpha}$ instances of $\mathcal{F}_{\mathrm{OT}}\left[\kappa^{\alpha}\right]$ with $p\left(\kappa^{\alpha}\right)$ invocations of a semi-honest stand-alone OT and communication cost $p\left(\kappa^{\alpha}\right)$, where $p(\cdot)$ is some polynomial, as long as $\alpha \leq 1 / 2$. We set $\alpha \in(0,1 / 2)$ such that $p\left(\kappa^{\alpha}\right)=o\left(\kappa^{c}\right)$.

Using Lemma 2 with parameters $\sigma=\kappa$, and $n, \epsilon$ such that $n^{1+\epsilon}=\kappa^{\alpha} / 10$, and $\ell=\kappa^{c}$, we can UC-realize $\mathcal{F}_{\mathrm{MCOM}}\left[\kappa^{c}\right]$ by making $n+2 n^{1-\epsilon}$ calls to $\mathcal{F}_{\mathrm{OT}_{\mathrm{R}}}^{\delta}\left[\kappa^{c} / n\right]$ with $\delta=\left(2 n^{\epsilon}+4\right)^{-1}$. Then, setting $\kappa_{\text {prg }}=\kappa^{\alpha}$, we use Lemma 1 to UC-realize these $n+2 n^{1-\epsilon}$ calls to $\mathcal{F}_{\mathrm{OT}_{\mathrm{R}}}^{\delta}\left[\kappa^{c} / n\right]$ with communication complexity $\left(n+2 n^{1-\epsilon}\right)$. $\left(\left(\kappa^{c} / n\right)+(1 / \delta)+3 \kappa^{\alpha} \cdot(1 / \delta)\right)$ while making $2 n^{1+\epsilon}+8 n$ calls to $\mathcal{F}_{\mathrm{OT}}\left[\kappa_{\mathrm{prg}}\right]$. 
Thus, for parameters $n, \epsilon, \kappa_{\text {prg }}$ as described above, we see that the communication complexity is $\kappa^{c}(1+o(1))$ while making (at most) $\kappa^{\alpha}$ calls to $\mathcal{F}_{\mathrm{OT}}\left[\kappa^{\alpha}\right]$. As described in the previous paragraph, these $\kappa^{\alpha}$ calls to $\mathcal{F}_{\mathrm{OT}}\left[\kappa^{\alpha}\right]$ can be implemented with communication cost $o\left(\kappa^{c}\right)$. Therefore, a single instance of $\mathcal{F}_{\mathrm{MCOM}}\left[\kappa^{c}\right]$ can be realized with communication cost $\kappa^{c}(1+o(1))$ in each phase.

For any setup where it is possible to construct UC-secure commitments on $\kappa$-bit strings (i.e., realize $\mathcal{F}_{\mathrm{MCOM}}[\kappa]$ ), then assuming the existence of semi-honest stand-alone oblivious transfer, Theorem 3 implies that it is possible to realize UC-secure commitments on strings of arbitrary length (in particular, on $\kappa$-bit strings) with rate $1-o(1)$ in that model. We explicitly state this for the CRS model, where it is known that a protocol for UC commitments in the CRS model implies the existence of semi-honest stand-alone oblivious transfer [15].

Corollary 1. If UC commitments exist in the CRS model, then they exist with rate $1-o(1)$.

\subsection{UC Commitment Length Extension Implies OT}

We now show that the existence of semi-honest stand-alone OT is necessary for the result above.

Theorem 4. Let $\kappa$ be a computational security parameter, and suppose there exists a protocol in which at most one party is allowed to make (at most) a single call to $\mathcal{F}_{\mathrm{MCOM}}[\kappa]$ to $U C$-realize a single instance of $\mathcal{F}_{\mathrm{MCOM}}[3 \kappa]$. Then there exists a protocol for semi-honest stand-alone OT.

Here we present only a proof sketch. The full proof is deferred to the full version.

Proof. We begin with a proof (sketch) for a weaker statement, namely, that UC commitment length extension from $\kappa$ bits to $3 \kappa$ bits implies key agreement. Recall that key agreement is implied by OT.

Key Agreement from Length Extension. Let $\Pi$ denote the commitment protocol assumed to exist. We construct a bit agreement protocol between two parties, $A$ and $B$, from $\Pi$ as follows:

- $A$ commits to a random $3 \kappa$-bit string $m$ by acting as the honest sender in an execution of $\Pi$, and in addition, sends the query $q \in\{0,1\}^{\kappa}$ it makes to the short commitment oracle and a random $r \in\{0,1\}^{\kappa}$;

- $\quad B$ runs the UC straight-line extractor for $\Pi$ to obtain $m$.

Both parties then agree on the Goldreich-Levin hard-core bit 20] $b=\langle m, r\rangle$ of $m$.

We now want to argue that an eavesdropper does not learn anything about $b$ in two steps:

- First, if we ignore the query $q$, then the view of the eavesdropper is exactly the commitment-phase transcript for $\Pi$, which reveals no information about $m$, which means $m$ has $3 \kappa$ bits of information-theoretic entropy. 
- The query $q$ then reveals at most $\kappa$ bits of information about $m$. Therefore, even upon revealing $q$, the message $m$ still has $\approx 2 \kappa$ bits of (min-)entropy. Then, the Goldreich-Levin hard-core bit works as a randomness extractor to derive a random bit from $m$.

Correctness is straightforward. To establish security against an eavesdropper, we crucially use the fact that a $\mathrm{UC}$ commitment scheme is equivocal, which allows us to essentially argue that $m$ has $3 \kappa$ bits of information-theoretic entropy. (Indeed, revealing $\kappa$ bits of information about a $3 \kappa$-bit pseudorandom string could reveal the entire string, as is the case when we reveal the seed used to generate the output of a pseduorandom generator.)

Remark. For technical reasons, we will require that the equivocal simulator can simulate not only the public transcript of the protocol, but also the query $q$ made to the short commitment oracle. The existence of such a simulator does not follow immediately from UC security, since the query $q$ may not be revealed to the malicious receiver and the environment. To handle this issue, we basically proceed via a case analysis:

- If the honest sender always reveals $q$ to the receiver either in the commit or the reveal phase, then the equivocal simulator must be able to simulate the query $q$ since it is part of the public transcript.

- Otherwise, we show by a simple argument that a cheating receiver can break the hiding property of the commitment scheme. (See full version for details.)

We are now ready to show the OT implication.

OT from Length Extension. In the OT protocol, $A$ holds $\left(b_{0}, b_{1}\right), B$ holds $\sigma$, and $B$ wants to learn $b_{\sigma}$. The protocol proceeds as follows:

- Alice runs two independent executions $\Pi_{0}, \Pi_{1}$ of the key agreement protocol for two random strings $m_{0}, m_{1} \in\{0,1\}^{3 \kappa}$ in parallel. In addition, $A$ sends

$$
z_{0}=b_{0} \oplus\left\langle m_{0}, r_{0}\right\rangle, \quad z_{1}=b_{1} \oplus\left\langle m_{1}, r_{1}\right\rangle .
$$

- In the execution $\Pi_{\sigma}, B$ behaves as in the key agreement protocol, which allows him to learn $\left\langle m_{\sigma}, r_{\sigma}\right\rangle$ and thus recover $b_{\sigma}$. In the other execution, $B$ acts as the honest receiver in an execution of commitment scheme $\Pi$.

Correctness follows readily from that of key agreement. We argue security as follows:

- First, we claim that a corrupted semi-honest $A$ does not learn $\sigma$. This follows from UC security of the commitment scheme against corrupted senders.

- Next, we claim that a corrupted semi-honest $B$ does not learn $b_{1-\sigma}$. This follows essentially from a similar argument to that for the security of the key agreement protocol with two notable differences: (i) in the execution $\Pi_{1-\sigma}$, $B$ acts as the honest receiver in $\Pi$ (instead of running the extractor as in the key agreement protocol), and (ii) a semi-honest $B$ learns the coin tosses of the receiver in $\Pi$, whereas an eavesdropper for the key agreementprotocol 
does not. Handling (i) is fairly straightforward albeit a bit technical; to handle (ii), we simply use the fact that the commitment phase transcript reveals no information about the committed value, even given the coin tosses of the honest receiver.

\section{References}

1. Abdalla, M., Benhamouda, F., Blazy, O., Chevalier, C., Pointcheval, D.: SPHFfriendly non-interactive commitments. In: Sako, K., Sarkar, P. (eds.) ASIACRYPT 2013, Part I. LNCS, vol. 8269, pp. 214-234. Springer, Heidelberg (2013)

2. Beaver, D.: Correlated pseudorandomness and the complexity of private computations. In: 28th Annual ACM Symposium on Theory of Computing (STOC), pp. 479-488. ACM Press (May 1996)

3. Bendlin, R., Damgård, I., Orlandi, C., Zakarias, S.: Semi-homomorphic encryption and multiparty computation. In: Paterson, K.G. (ed.) EUROCRYPT 2011. LNCS, vol. 6632, pp. 169-188. Springer, Heidelberg (2011)

4. Blazy, O., Chevalier, C., Pointcheval, D., Vergnaud, D.: Analysis and improvement of Lindell's UC-secure commitment schemes. In: Jacobson, M., Locasto, M., Mohassel, P., Safavi-Naini, R. (eds.) ACNS 2013. LNCS, vol. 7954, pp. 534-551. Springer, Heidelberg (2013)

5. Brassard, G., Crepeau, C., Robert, J.-M.: Information theoretic reduction among disclosure problems. In: FOCS, pp. 168-173 (1986)

6. Canetti, R.: Universally composable security: A new paradigm for cryptographic protocols. In: 42nd Annual Symposium on Foundations of Computer Science (FOCS), pp. 136-145. IEEE (October 2001)

7. Canetti, R., Fischlin, M.: Universally composable commitments. In: Kilian, J. (ed.) CRYPTO 2001. LNCS, vol. 2139, pp. 19-40. Springer, Heidelberg (2001)

8. Canetti, R., Lindell, Y., Ostrovsky, R., Sahai, A.: Universally composable twoparty and multi-party secure computation. In: 34th Annual ACM Symposium on Theory of Computing (STOC), pp. 494-503. ACM Press (May 2002)

9. Choi, S.G., Dachman-Soled, D., Malkin, T., Wee, H.: Simple, black-box constructions of adaptively secure protocols. In: Reingold, O. (ed.) TCC 2009. LNCS, vol. 5444, pp. 387-402. Springer, Heidelberg (2009)

10. Choi, S.G., Katz, J., Wee, H., Zhou, H.-S.: Efficient, adaptively secure, and composable oblivious transfer with a single, global CRS. In: Kurosawa, K., Hanaoka, G. (eds.) PKC 2013. LNCS, vol. 7778, pp. 73-88. Springer, Heidelberg (2013)

11. Crépeau, C.: Equivalence between two flavours of oblivious transfers. In: Pomerance, C. (ed.) CRYPTO 1987. LNCS, vol. 293, pp. 350-354. Springer, Heidelberg (1988)

12. Damgård, I., David, B., Giacomelli, I., Nielsen, J.B.: Homomorphic uc commitments in uc (2013) (manuscript)

13. Damgård, I., Groth, J.: Non-interactive and reusable non-malleable commitment schemes. In: 35th Annual ACM Symposium on Theory of Computing (STOC), pp. 426-437. ACM Press (June 2003)

14. Damgård, I., Nielsen, J.B.: Perfect hiding and perfect binding universally composable commitment schemes with constant expansion factor. In: Yung, M. (ed.) CRYPTO 2002. LNCS, vol. 2442, pp. 581-596. Springer, Heidelberg (2002)

15. Damgård, I., Nielsen, J.B., Orlandi, C.: On the necessary and sufficient assumptions for UC computation. In: Micciancio, D. (ed.) TCC 2010. LNCS, vol. 5978, pp. 109-127. Springer, Heidelberg (2010) 
16. Fischlin, M., Libert, B., Manulis, M.: Non-interactive and reusable universally composable string commitments with adaptive security. In: Lee, D.H., Wang, X. (eds.) ASIACRYPT 2011. LNCS, vol. 7073, pp. 468-485. Springer, Heidelberg (2011)

17. Franklin, M., Yung, M.: Communication complexity of secure computation. In: STOC, pp. 699-710 (1992)

18. Frederiksen, T., Jakobsen, T., Nielsen, J., Nordholt, P., Orlandi, C.: Minilego: Efficient secure two-party computation from general assumptions. In: Johansson, T., Nguyen, P.Q. (eds.) EUROCRYPT 2013. LNCS, vol. 7881, pp. 537-556. Springer, Heidelberg (2013)

19. Fujisaki, E.: A framework for efficient fully-equipped UC commitments. ePrint 2012/379 (2012)

20. Goldreich, O., Levin, L.A.: A hard-core predicate for all one-way functions. In: 21st Annual ACM Symposium on Theory of Computing (STOC), pp. 25-32. ACM Press (May 1989)

21. Groth, J., Sahai, A.: Efficient non-interactive proof systems for bilinear groups. In: Smart, N.P. (ed.) EUROCRYPT 2008. LNCS, vol. 4965, pp. 415-432. Springer, Heidelberg (2008)

22. Hofheinz, D., Müller-Quade, J., Unruh, D.: On the (im-)possibility of extending coin toss. In: Vaudenay, S. (ed.) EUROCRYPT 2006. LNCS, vol. 4004, pp. 504-521. Springer, Heidelberg (2006)

23. Ishai, Y., Kilian, J., Nissim, K., Petrank, E.: Extending oblivious transfers efficiently. In: Boneh, D. (ed.) CRYPTO 2003. LNCS, vol. 2729, pp. 145-161. Springer, Heidelberg (2003)

24. Ishai, Y., Prabhakaran, M., Sahai, A.: Founding cryptography on oblivious transfer - efficiently. In: Wagner, D. (ed.) CRYPTO 2008. LNCS, vol. 5157, pp. 572-591. Springer, Heidelberg (2008)

25. Jutla, C.S., Roy, A.: Shorter quasi-adaptive nizk proofs for linear subspaces. In: Sako, K., Sarkar, P. (eds.) ASIACRYPT 2013, Part I. LNCS, vol. 8269, pp. 1-20. Springer, Heidelberg (2013)

26. Kilian, J.: Founding cryptography on oblivious transfer. In: STOC, pp. 20-31 (1988)

27. Kraschewski, D.: Complete primitives for information-theoretically secure twoparty computation (2013), http://digbib.ubka.uni-karlsruhe.de/volltexte/ 1000035100 (retrieved October 14, 2013)

28. Lindell, Y.: Highly-efficient universally-composable commitments based on the DDH assumption. In: Paterson, K.G. (ed.) EUROCRYPT 2011. LNCS, vol. 6632, pp. 446-466. Springer, Heidelberg (2011)

29. Lindell, Y., Zarosim, H.: On the feasibility of extending oblivious transfer. In: Sahai, A. (ed.) TCC 2013. LNCS, vol. 7785, pp. 519-538. Springer, Heidelberg (2013)

30. Maji, H., Prabhakaran, M., Rosulek, M.: Cryptographic complexity classes and computational intractability assumptions. In: ICS, pp. 266-289 (2010)

31. Nielsen, J., Nordholt, P., Orlandi, C., Burra, S.S.: A new approach to practical active-secure two-party computation. In: Safavi-Naini, R., Canetti, R. (eds.) CRYPTO 2012. LNCS, vol. 7417, pp. 681-700. Springer, Heidelberg (2012)

32. Nielsen, J.B.: Separating random oracle proofs from complexity theoretic proofs: The non-committing encryption case. In: Yung, M. (ed.) CRYPTO 2002. LNCS, vol. 2442, pp. 111-126. Springer, Heidelberg (2002)

33. Nishimaki, R., Fujisaki, E., Tanaka, K.: An eficient non-interactive universally composable string-commitment scheme. IEICE Transactions, 167-175 (2012) 
34. Peikert, C., Vaikuntanathan, V., Waters, B.: A framework for efficient and composable oblivious transfer. In: Wagner, D. (ed.) CRYPTO 2008. LNCS, vol. 5157, pp. 554-571. Springer, Heidelberg (2008)

\section{A Efficient Commitments for Cut-and-Choose}

While our rate 1 construction has good concrete efficiency for large string commitments, the case of short string commitments leaves a lot to be desired. An obvious approach to handle short strings is simply to concatenate these strings together to form one large string, and then use the rate 1 construction with this string as the input message. While this approach does provide a concrete rate close to 1 when the number of instances is large, it has the drawback that all instances of short strings must be opened simultaneously. In this section, we design more efficient commitment scheme for handling multiple instances of $\kappa$-bit strings with two opening phases (as required in techniques such as cut-and-choose). The extension to three or more opening phases is straightforward.

For $i \in[n]$, let the $i$-th $\kappa$-bit string be denoted by $m_{i}$, and let $m=\left(m_{1}, \ldots, m_{n}\right)$. Let $p$ denote the number of opening phases, and for $j \in[p]$, let $u_{j}$ denote the characteristic vector of the subset $S_{j} \subseteq[n]$ of the strings that need to opened in the $j$-th opening phase. Note that $u_{j}$ is not known to the sender during the commit phase.

Our high level idea is as follows. As in our rate 1 construction, we let the sender encode $m$ in to $m^{\prime}$ using the rate 1 encoding scheme. In addition, for each $i \in[p]$, the sender uses the rate $1 / 2$ encoding scheme (naturally derived from Enc) to encode the zero string $(0, \ldots, 0) \in \mathbb{F}^{n}$ twice using independent randomness to obtain codewords $z^{(1)}, z^{(2)}$ (each of length $2 n^{\prime}$ ). Next the sender prepares to send symbols through the Rabin-OT oracle. For this, it constructs $M_{k}=$ $\left(m_{k}^{\prime}, z_{k}^{(1)}, z_{k}^{(2)}\right)$ for $k \leq n^{\prime}$, and symbols $M_{k}=\left(z_{k}^{(1)}, z_{k}^{(2)}\right)$ for $k \in\left\{n^{\prime}+1, \ldots, 2 n^{\prime}\right\}$, as the $k$-th input to the Rabin-OT oracle. Then, it transmits $M_{k}$ through RabinOT oracle with parameter $\delta^{\prime}=\delta / 2$ (where $\delta$ is the best parameter for obtaining commitments on strings of length $n \kappa)$. Then, in the $j$-th opening phase, the receiver sends the randomness (alternatively, a seed to a PRG) to encode $u_{j}$ into $u_{j}^{\prime}$ using the rate 1 encoding scheme. Now, denote the underlying polynomials (cf. Figure 4) for (1) the rate 1 encoding of $m$ by $q_{m},(2)$ the rate $1 / 2$ encoding of $z^{(j)}$ as $q_{z}^{(j)}$, and (3) the rate 1 encoding of $u_{j}$ by $q_{u}^{j}$. In the $j$-th opening phase, the sender simply reveals the polynomial $q^{(j)}=\left(q_{m} \cdot q_{u}^{j}\right)+q_{z}^{(j)}$. Now, let $\left\{\tilde{M}_{k}\right\}_{k \in J}$ denote the messages received by the receiver. The receiver checks if for all $k \in$ $J \cap\left[n^{\prime}\right]$, it holds that $\tilde{M}_{k}=\left(\tilde{m}_{k}^{\prime}, \tilde{z}_{k}^{(1)}, \tilde{z}_{k}^{(2)}\right)$ satisfies $q^{(j)}(k)=\left(\tilde{m}_{k}^{\prime} \cdot q_{u}^{j}(k)\right)+\tilde{z}_{k}^{(j)}$. If the check succeeds, then the receiver computes $v_{i}=q^{(j)}\left(e_{i}\right)$, where $e_{i}$ are the publicly known points as described in Figure 4. If for all $i \notin S_{j}$, it holds that $v_{i}=0$, then receiver outputs $\left\{v_{j}\right\}_{j \in S_{j}}$ and terminates, else it outputs $\perp$ and terminates. Let $c_{1}, c_{2}, c_{3}$ represent our concrete cost of realizing commitments on strings of length $n \kappa$ in the offline, the online commit, and the online reveal phases respectively. It can be verified that the cost of the above scheme that implements $n$ instances of $\kappa$-bit commitments with two opening phases is $\approx 8 c_{1}, 2 c_{2}, 2 c_{3}$ in the offline, the online commit, and the online reveal phases respectively. 